\title{
Short-Branched Fluorinated Polyurethane Coating Exhibiting Good Comprehensive Performance and Potential UV Degradation in Leather Waterproofing Modification
}

\author{
Shouhua Su, Juan Wang, Chao Li, Jinfeng Yuan, Zhicheng Pan and Mingwang Pan *(D)
}

check for

updates

Citation: Su, S.; Wang, J.; Li, C.; Yuan,

J.; Pan, Z.; Pan, M. Short-Branched

Fluorinated Polyurethane Coating

Exhibiting Good Comprehensive

Performance and Potential UV

Degradation in Leather

Waterproofing Modification. Coatings

2021, 11, 395. https://doi.org/

10.3390/coatings11040395

Academic Editor: Mohor Mihelčič

Received: 11 February 2021

Accepted: 27 March 2021

Published: 30 March 2021

Publisher's Note: MDPI stays neutral with regard to jurisdictional claims in published maps and institutional affiliations.

Copyright: (c) 2021 by the authors. Licensee MDPI, Basel, Switzerland. This article is an open access article distributed under the terms and conditions of the Creative Commons Attribution (CC BY) license (https:/ / creativecommons.org/licenses/by/ $4.0 /$ )
Hebei Key Laboratory of Functional Polymers, School of Chemical Engineering and Technology, Hebei University of Technology, Tianjin 300130, China; shouhuasu@163.com (S.S.); juanwang117@163.com (J.W.); chaoli1129@163.com (C.L.); yuanjf@hebut.edu.cn (J.Y.); panz@hebut.edu.cn (Z.P.)

* Correspondence: mwpan@126.com

Abstract: In the current leather market, waterproof leather occupies a large proportion, where waterproofness has become one of the important standards for leather selection. However, the most advanced fluorine-containing waterproofing agents on the market always have long chains of over eight carbons (C8), whose use has been restricted due to their bioaccumulation and recalcitrance in natural environment. Consequently, creating waterproof materials characterized by their environmentally friendly qualities and high performance is of great significance. Herein, we report a novel strategy for preparation of the fluorinated polyurethanes containing short branched fluorocarbon chains, and apply it in leather waterproofing. Because the fluorine-containing chain segments are enriched on the coating surface, the waterproof agent coating shows good hydrophobicity, low water absorption, high wear resistance and potential photodegradation of performances. Additionally, the water and oil proof performances of the coating are comparable to that of the marketed C8 waterproofing agent. Its solvent-resistant and antifouling performances are also outstanding. Therefore, the coating can meet the property requirements for daily use and has broad application prospects.

Keywords: short branched fluorocarbon chain; fluorinated polyurethane (FPU); waterproof coating; leather surface modification

\section{Introduction}

At present, leather has a wide range of applications, but its development is limited by its easy aging and poor properties in terms of water proofing and scratch resistance. The main way to improve the performance of leather materials is to coat its surface with a layer of protective film. The common coatings on the market can roughly be divided into three categories, namely polyurethane [1-6], polyacrylate [7-9], and organic silicon [10-12]. Among them, polyurethane (PU) is the first reported and widely studied due to its strong structural designability and excellent wear resistance [13]. However, the waterproof performance of PU material itself is not enough to meet the requirements of leather materials in some special fields, such as in a long term exposure to a humid environment. To further improve the waterproof, oil-resistant and anti-smudge properties of PU coatings, fluorinated polyurethane (FPU) waterproofing agent is prepared often by introducing fluorine-containing groups into the PU molecular structure. On the one hand, the FPU retains the special structural characteristics of PU itself, which can bring lots of excellent properties to the material, for example good film formation, wear resistance and toughness. On the other hand, the introduction of fluorine-containing segments endows polyurethane-based materials with other special properties (like excellent waterproof, oilproof and anti-smudge properties [14-22]) due to its low surface energy [23], which determines that the FPU will have better application prospects in more fields than the traditional PU. The introduction of long-chain perfluoroalkyl (LF) groups to PU is currently 
the most used method for FPU preparation through implanting fluorinated polyols as soft chain segments [24,25], perfluorinated alcohols as end sealants [26,27], or fluorinated compounds with multi-functional groups as chain extenders [28-30].

To date, fluorinated chemicals containing LF groups [31], the most advanced waterproofing agent on the market, have been verified, demonstrating that their manufacture originated from straight-chain C8 perfluoroalkyl sulfonic acid or perfluoroalkyl acids, such as C8 fluorocarbons, perfluorooctane sulfonic acid (PFOS) and perfluorooctanoic acid (PFOA) that have potential biotoxicity like bioaccumulation and environmental persistence [32-36]. In view of these worrying dangers, many countries, such as Canada, the United States and China, have adopted certain regulatory measures to prohibit its use in the relevant industrial production. Fortunately, compounds with shorter fluorocarbon (SF) chains are thought to be significantly less toxic, and the subsequent breakdown products are innoxious $[37,38]$. Although FPU with SF components is more environmentally friendly than FPU with LF components, its hydrophobic, oleophobic and anti-smudge properties are significantly worse than those of long-chain FPU. Thus, it is particularly important to explore a new type of fluorinated polyurethane that is not only environmentally friendly but also has outstanding coating properties in the leather field. As for FPU with short branched fluorocarbon chains, it is unclear whether it can satisfy the above two conditions at the same time, and there have been few reports on this to the best of our knowledge. Therefore, research into the fluorinated polyurethane with short branched chains in the leather field will have strong theoretical and practical significance.

In this work, a novel type of short-branched fluorinated PU was prepared by first synthesizing short-branched chain fluorinated alcohol and then implanting it into the PU structure as an end-capping agent. As a main raw material, hexafluoropropylene trimer is not restricted in use [39]. Thus, we initially synthesized a fluoro-alcohol by reacting hexafluoropropylene trimer with p-hydroxybenzyl alcohol (Figure 1ai) [40]. Next, a new FPU waterproofing agent was prepared by pre-reaction of 1,4-butanediol and toluene diisocyanate, successively end-capping with fluoro-alcohol $\left(\mathrm{R}_{\mathrm{f}}-\mathrm{OH}\right)$ and ethanol respectively (Figure 1 aii). We adjusted the fluorine content in the final mixture by changing the molar ratio of ethanol and fluoro-alcohol, and maintained a molar ratio of approximately 1:1 between isocyanate groups and hydroxy groups. Fluorine-containing short branched chains not only provide FPU with excellent surface performance, low water absorption, and satisfied wear resistance, they also provide it with potential photodegradation. Most notably, the FPU could be compared to the commercial C8 waterproofing agent, and the former also exhibited superior waterproof, oilproof and antifouling properties, prominent solvent resistance, and potential photocatalytic degradation.

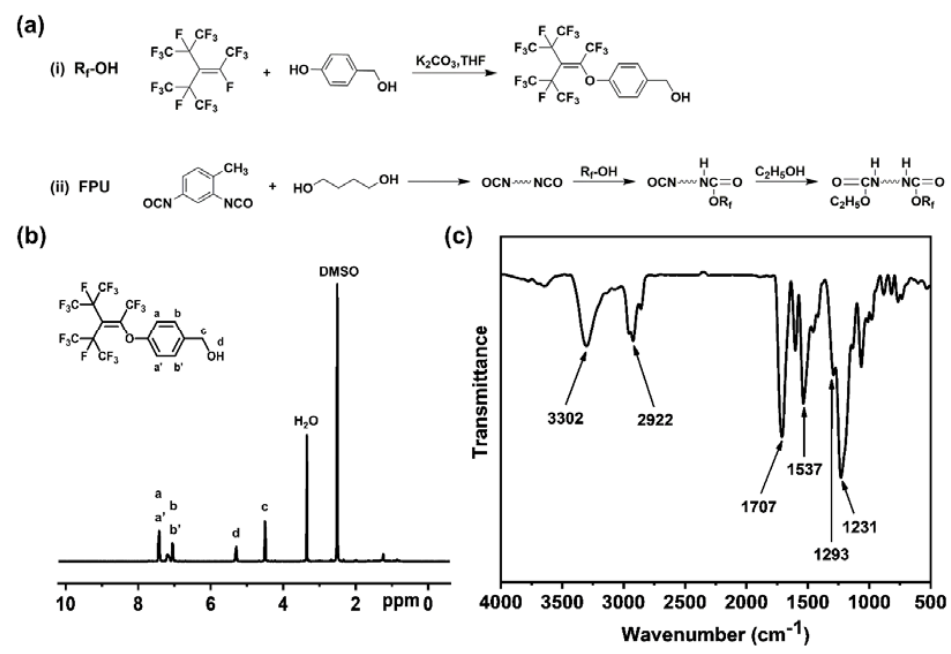

Figure 1. (a) Preparation procedure of (i) $\mathrm{R}_{\mathrm{f}}-\mathrm{OH}$ and (ii) FPU; (b) $1 \mathrm{H}-\mathrm{NMR}$ spectrum of $\mathrm{R}_{\mathrm{f}}-\mathrm{OH}$ in DMSO-d6; (c) FTIR spectrum of FPU. 


\section{Materials and Methods}

\subsection{Materials}

Hexafluoropropylene trimer (HFPT) was purchased from Quzhou Chemical Industry Co., Ltd. (Quzhou, China). p-Hydroxybenzyl alcohol, potassium carbonate $\left(\mathrm{K}_{2} \mathrm{CO}_{3}\right)$, tetrahydrofuran (THF), toluene diisocyanate (TDI), 1,4-butanediol (BDO), N,N-dimethylformamide (DMF) and ethanol $\left(\mathrm{C}_{2} \mathrm{H}_{5} \mathrm{OH}\right)$ were supplied by Aladdin Chemical Reagent Co., Ltd. (Shanghai, China). C8 waterproofing agent was provided by Taifu Chemical Technology Co., Ltd. (Shanghai, China).

\subsection{Synthesis of $\mathrm{R}_{f}-\mathrm{OH}$}

p-Hydroxybenzyl alcohol (6.20 g) and $\mathrm{K}_{2} \mathrm{CO}_{3}(6.91 \mathrm{~g})$ were dissolved in $60 \mathrm{~mL}$ THF. HFPT (33.75 g) was added dropwise into a four-mouth flask under nitrogen atmosphere, followed by stirring and reflux for $4 \mathrm{~h}$ at $60{ }^{\circ} \mathrm{C}$. Afterwards the mixture was slowly cooled to room temperature and THF was removed with rotary evaporation to obtain a yellow liquid (20.78 g, 70\% yield).

\subsection{Synthesis of FPU}

BDO and DMF were mixed in a four-mouth flask at room temperature and then TDI was added dropwise into the reaction flask under a nitrogen atmosphere. The reaction system was kept at $70^{\circ} \mathrm{C}$ for $2 \mathrm{~h}$, then polyurethane prepolymer with two $\mathrm{NCO}$ groups at the ends was prepared. Next, fluoro-alcohol $\left(\mathrm{R}_{\mathrm{f}}-\mathrm{OH}\right)$ was added to the system as one capping agent, and the reaction was also maintained for $2 \mathrm{~h}$. Finally, ethanol was added as another capping agent under continuous stirring for another $2 \mathrm{~h}$. Thus, the FPU at one end of a molecular chain was sealed with fluoro-alcohol and the other end was successfully sealed with ethanol. In this synthesis stage, we adjusted the fluorine content in the final mixture by changing the molar ratio between ethanol (ET) and fluoro-alcohol (FA) and maintained a molar ratio of approximately 1:1 between isocyanate and hydroxyl groups.

\subsection{Leather Waterproof Coating}

The leather surface was wiped with an absorbent cotton before coating the FPU waterproofing agent, then a very small amount of FPU solution was dropped and rollcoated on the leather surface with a clean glass rod. Finally, the treated leather was cured at $130{ }^{\circ} \mathrm{C}$ for $20 \mathrm{~min}$.

\subsection{Characterization and Measurements}

\subsubsection{Chemical Structure Analysis}

For $\mathrm{R}_{\mathrm{f}}-\mathrm{OH}, 1 \mathrm{H}$ NMR spectrum was obtained on Bruker 400 nuclear magnetic resonance spectrometer and HRMS-ESI was obtained on Bruker Compact Q-TOF. The composition of FPU coating was analyzed by Fourier transform infrared spectroscopy (FTIR). FTIR spectrum was recorded using a FTIR spectrometer (Tensor-27, Bruker, Karlsruhe, Germany) at room temperature. The mixture of solid sample and desiccative potassium bromide $(\mathrm{KBr})$ was pressed into pellets for FTIR research.

\subsubsection{Surface Element Analysis}

The contents of chemical elements on the surfaces of PU and FPU coatings were detected by X-ray photoelectron spectroscopy (XPS, ESCALAB 250 Xi, Thermo Fisher Scientific, Waltham, MA, USA). The elements of the coatings and their contents were also studied by energy-dispersive spectrometer (EDS), which was performed on a scanning electron microscope (FEI Nova Nano SEM 450) operating at $10 \mathrm{kV}$. To avoid charge-loading, the sample surface was sputtered with a thin layer of gold before the EDS experiment.

\subsubsection{Wetting Test}

Static water drop contact angle (WCA) and surface energy of the FPU membrane were tested by DSA $30 \mathrm{~S}$ apparatus (Krüss Co., Hamburg, Germany) with $10 \mu \mathrm{L}$ of water at room 
temperature. The WCA was the mean value of five measurements for water droplets at different places on each sample surface.

\subsubsection{Water Absorption}

The FPU solution was uniformly sprayed on a glass plate with an area of $2.54 \times 7.62 \mathrm{~cm}^{2}$, and then dried and cured at $130^{\circ} \mathrm{C}$ to obtain a FPU coating film with a thickness of $80 \mu \mathrm{m}$. The sample was placed into distilled water at $25^{\circ} \mathrm{C}$ for $120 \mathrm{~h}$. During this soaking process, it was taken out at certain intervals, the water on its surface was quickly wiped with filter paper, and then the weight was measured. The water absorption was calculated by following Formula (1):

$$
w=\frac{m_{2}-m_{1}}{m_{1}} \times 100 \%
$$

where $m_{1}$ is the mass of the coating before being put into the water, $m_{2}$ is the mass of the coating after being put into the water, and $w$ is water absorption.

\subsubsection{Abrasion Analysis}

Quantitative wear analysis was carried out using a JM-IV wear tester with two abrasive CS-10 wheels. The grinding wheel with each load of $250 \mathrm{~g}$ was used to conduct a wear test on the leather treated with the FPU waterproof agent. The contact pressure of the coating film was estimated to be about $12.1 \mathrm{MPa}$, and the leather was subjected to uniform stress in the slow and uniform rotation of the grinding wheel.

\subsubsection{Potential UV Degradation}

UV light irradiation was used to investigate the potential degradation performance of the FPU waterproofing agent. The leather treated with the FPU waterproofing agent was placed under a $365 \mathrm{~nm}$ UV lamp with a power of $30 \mathrm{~W}$, which was located $0.14 \mathrm{~m}$ from the light source.

\subsubsection{Waterproof Grade Test}

According to BS ISO 23232:2009, the dripping test method was adopted. According to different surface tensions of isopropanol/water solution with different volume fractions, the waterproofing grades are divided into $0 \sim 8$ grades, which successively increase from 0 to 8 , as shown in Table S1 in the Supporting Information. During this test, $5 \mu \mathrm{L}$ of the liquid was dropped on the leather surface treated with the FPU waterproofing agent at an interval of $5 \mathrm{~mm}$. If the surface of leather sample was not wetted after placement for $10 \mathrm{~s}$, in which the profile morphology and contact angle of the droplet did not significantly change, the waterproof grade was passed. Afterwards, the leather was then tested with a higher grade of water-isopropyl alcohol mixture until the surface was wetted. The final pass grade was the waterproof grade of FPU coated leather.

\subsubsection{Oilproof Grade Test}

According to BS ISO 14419:2010, oilproof grades can be divided into 1 8 grades on the basis of different types of oil with different surface tensions (Table S2 in the Supporting Information). During this test, $5 \mu \mathrm{L}$ of oil (analytical reagent) was dropped onto the FPUcoated leather surface in a spacing of $5 \mathrm{~mm}$. If the sample surface was not wetted after $10 \mathrm{~s}$, the profile shape and contact angle of the droplet had no obvious change to suggest the waterproof level pass. Then, the higher grade of oil reagent was used to test until the leather surface was wetted. As a result, the final approved grade was the oil proof grade of the FPU modified leather.

\subsubsection{Anti-Smudge Performance}

The anti-smudge performance of our designed coating was further investigated. We dropped the ink on the sloping leather to observe the flow mark of the ink print. 


\subsubsection{Solvent Resistance}

According to GB/T 23989-2009, the absorbent cotton was immersed in xylene solvent to obtain a wetted state (no liquid drops should be dropped when it was extruded by hand). Subsequently, using a safe protection, the index finger and thumb clamped the center of the absorbent cotton at a $45^{\circ}$ tilt angle against the coating surface. A total of 25 forward and backward wipes were performed at a proper pressure to evaluate the solvent resistance of the coating.

\section{Results}

\subsection{Chemical Structure Analyses}

The successful preparation of $\mathrm{R}_{\mathrm{f}}-\mathrm{OH}$ was confirmed by $1 \mathrm{H}$ NMR and HRMS-ESI. Figure $1 \mathrm{~b}$ shows the $1 \mathrm{H}$ NMR spectrum $\left(400 \mathrm{MHz}\right.$, DMSO-d6) of $\mathrm{R}_{\mathrm{f}}-\mathrm{OH}$ sample: $\mathrm{c}$, chemical shift $4.50 \mathrm{ppm}(\mathrm{s}, 2 \mathrm{H}) ; \mathrm{d}, 5.29 \mathrm{ppm}(\mathrm{s}, 1 \mathrm{H}) ; \mathrm{b}$ and $\mathrm{b}^{\prime}, 7.02 \sim 7.23 \mathrm{ppm}(\mathrm{m}, 2 \mathrm{H})$; a and $\mathrm{a}^{\prime}$, 7.37 7.47 ppm (m, 2H). HRMS-ESI $(m / z)$ : cacld for $\mathrm{C}_{16} \mathrm{H}_{7} \mathrm{~F}_{17} \mathrm{O}_{2} \mathrm{Na}[\mathrm{M}+\mathrm{Na}]^{+}$577.0066, found 577.0061 .

To identify the chemical components of FPU coating, its FTIR measurement spectrum was recorded, as is given in Figure 1c, where the characteristic peaks in $\mathrm{N}-\mathrm{H}\left(3302 \mathrm{~cm}^{-1}\right)$, $\mathrm{C}=\mathrm{O}\left(1707 \mathrm{~cm}^{-1}\right)$, and saturated $\mathrm{C}-\mathrm{H}\left(2922 \mathrm{~cm}^{-1}\right)$ stretching vibrations can be observed. The absorption band at $1537 \mathrm{~cm}^{-1}$ resulted from N-H bending vibration of secondary amide. The disappearance of absorption bands of TDI's NCO group $\left(2270 \mathrm{~cm}^{-1}\right)$ and alcohol hydroxyl group $\left(3340 \mathrm{~cm}^{-1}\right)$ proved the successful synthesis of FPU oligomer [41]. However, the C-F stretching vibration absorption was not conspicuous as it might be overlapped by the strong absorption of C-O-C group at around $1231 \mathrm{~cm}^{-1}$, and a weak peak at $1293 \mathrm{~cm}^{-1}$ may be also due to the C-F stretching vibration. These results confirmed the chemical structure of FPU oligomer.

\subsection{Coating Surface Chemistry}

XPS is an analysis technique for detecting the surface chemistry of a material in the original state or after some treatment. The surface chemical composition of PU $(\mathrm{ET}: \mathrm{FA}=1: 0 \mathrm{~mol} / \mathrm{mol})$ and FPU $(\mathrm{ET}: \mathrm{FA}=0.5: 0.5 \mathrm{~mol} / \mathrm{mol}$ ) coatings were confirmed by XPS, as exhibited in Figure 2i. The surface atomic characteristic signals $\mathrm{C} 1 \mathrm{~s}(276.8 \mathrm{eV}), \mathrm{O}$ $1 \mathrm{~s}(524.4 \mathrm{eV}), \mathrm{N} 1 \mathrm{~s}(392.3 \mathrm{eV})$ were observed, while the peak at $682.4 \mathrm{eV}$ assigned to $\mathrm{F}(1 \mathrm{~s})$ was clearly observed only in the FPU sample. This indicated that F atoms were presented smoothly on the surface of the coating.

Generally speaking, fluorinated segments prefer to migrate to the material surface, which contributed to the low surface energy and hydrophobic property [42,43]. When the leather surface was covered with a uniform FPU waterproofing agent, the fluorinecontaining chain segments would migrate and enrich onto the surface during the drying process. Here, the XPS detection result of FPU (ET:FA $=0.5: 0.5)$ coating indicated that the fluorine content $(31.03 \mathrm{wt} \%)$ on its surface was much higher than the theoretically calculated content $(20.6 \mathrm{wt} \%)$, which clearly confirmed the enrichment effect of fluorinecontaining chain segments on the coating surface. The proposed mechanism for dynamic behavior of perfluoroalkyl segments during drying and curing is shown in Figure 2ii. As seen in Figure 2iii, the surface energy of the fluorine-free coating film was $57.61 \mathrm{mN} / \mathrm{m}$, while the surface energy of the coating with the incorporation of 0.25 molar ratio of FA decreased to $30.05 \mathrm{mN} / \mathrm{m}$. As the addition proportion of FA increased, the surface energy of the coating decreased continuously. Intriguingly, after the ET/FA molar ratio reached $0.5 / 0.5$, the surface energy was no longer significantly reduced with the increase in FA proportion. The explanation for this is that at a higher $\mathrm{F}$ element content, more fluorine migrated to the surface, meaning that the FPU waterproof coating had a lower surface energy until close to the saturated fluorine content on the surface. 

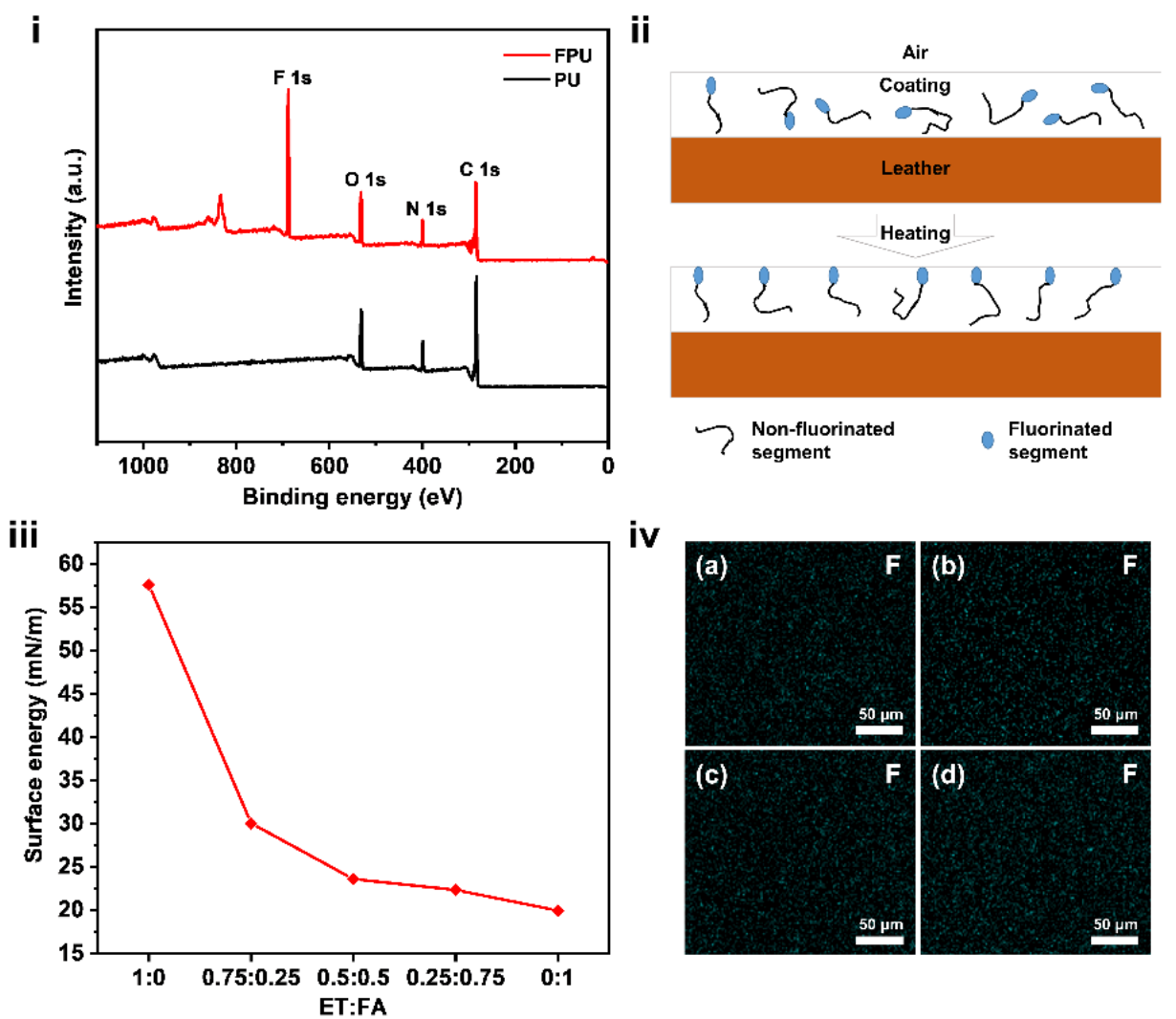

Figure 2. (i) XPS spectra of surface survey of pure PU (ET:FA = 1:0) coating and FPU coating (ET:FA = 0.5:0.5); (ii) Schematic representation of surface enrichment of fluorinated segments on the coating during the drying and curing process; (iii) Surface energy of the coatings against molar feed proportions of ET:FA; (iv) Fluorine atom (F) EDS mappings of the FPU coatings at different molar feed proportions of ET:FA. (a) 0.75:0.25, (b) 0.5:0.5, (c) 0.25:0.75, (d) 0:1.

To further confirm the change in fluorine-containing chain segments on the coating surface, EDS mapping of different ET:FA molar proportions of coatings was performed to further analyze the composition and content of elements on the coating surface. Figure 2iv showed the fluorine atom-based (F) EDS mappings of the FPU samples. It can be seen that the uniform distribution of $\mathrm{F}$ element on the surface of the whole coating could clearly be observed, and fluorine content increased significantly with the increase in FA feed proportion. As a result, it is the enrichment of fluorine-containing segments on the surface of materials that made the coating exhibit an outstanding waterproofing performance, which is the main reason it is unique to other materials.

\subsection{Surface Wettability}

Water droplet contact angle measurement is one of the most common methods used to evaluate surface waterproofing performance. As a direct reflection of the surface energy of the materials, the WCA has great guiding significance for the application of waterproof materials. The smaller WCA is, the better the wettability of liquid on a solid surface is. Consequently, contact angle can be used to measure the wettability. When WCA is less than $90^{\circ}$, the liquid can moisten the solid surface. The change in WCAs of the PU and FPU coatings with increasing FA proportion is given in Figure $3 \mathrm{a}$. It can be seen from Figure $3 a$ that the coating WCA increased with the increase in FA addition ratio. Compared with the pure PU (i.e., ET:FA $\left.=1: 0,75^{\circ}\right)$, the WCA of FPU coating with ET:FA $=0: 1\left(117^{\circ}\right)$ increased by $42^{\circ}$. In addition, the test result of WCA was similar to that of commercial C8 waterproofing agent. As a result, a hydrophobic surface was already formed due to the enrichment of fluorine-containing chain segments on the surface. Nevertheless, the 
increase in WCA was not obvious with the ET:FA ratio increasing up to 0.5:0.5, which may be due to the above-mentioned "saturation" of fluorine atoms on the film surface.
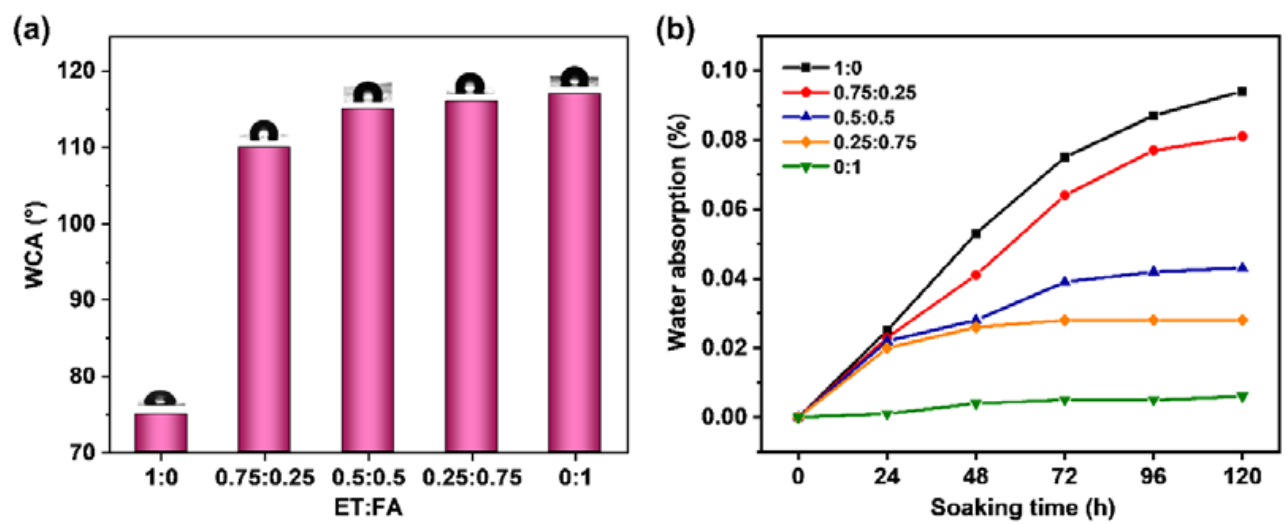

Figure 3. (a) Water drop contact angle (WCA) and (b) water absorption of waterproof coatings with different molar proportions of ET:FA.

Water resistance is also an important investigation index for the practical application of a waterproofing agent. The easy migration of fluorine-containing groups could cover the surface of the coating to reduce the water absorption. As a result, the water absorption for the FPU sample was smaller than the pure PU due to the existence of fluoride chain segments. In addition, the surface of the coating was more compact as the proportion of FA end-sealant increased, which made water molecules' penetration become more difficult. From Figure $3 b$, after $120 \mathrm{~h}$ of continuous immersion, the water absorption rate of PU without FA was $0.094 \%$, whereas the water absorption rates of FPU coatings incorporating FA gradually decreased with the increase in the proportion of FA addition. In detail, the water absorption rates of the FPU coatings at ET:FA of 0.5:0.5 and 0.25:0.75 were 0.048\% and $0.028 \%$, respectively, which was lower than half of PU. The FPU coatings showed the outstanding water resistance, and its water absorption rate was at a minimum when ET:FA molar ratio was 0:1, which was almost unaffected by the soaking time. This is also due to the low surface energy of fluorine-containing chain segments migration and enrichment onto the surface. Furthermore, the water absorption did not further change with the extension of soaking time and there is no shedding or foaming phenomenon on the coating surface, which indicated the excellent water resistance of FPU waterproof coating.

\subsection{Abrasion Resistance}

Abrasion resistance is an important performance index for practical applications of polymer coating. In this work, the waterproofing agent coating showed a satisfactory abrasion resistance. As illustrated in Figure 4a, wearing was tested by a taber abraser at a loading pressure of $12.1 \mathrm{MPa}$, and the experimental results displayed that the FPU-coated leather still maintained the hydrophobicity even after 600 abrasion cycles (Figure 4b). The coating WCAs increased with the increase in wear time. Among them, the WCA of FPU coating with a large proportion of FA incorporation exceeded $120^{\circ}$ after several hundred times of friction. It could be reasonably speculated that this interesting result was ascribed to the joint effects of roughness increase and the enrichment of fluorine-containing segments on the surface of the FPU waterproofing coating. When the number of wear times reached 700, the coating WCA decreased dramatically, no longer exhibiting the hydrophobic performance. This phenomenon can be attributed to the substantive damage of FPU coating on the leather surface. 
(a)

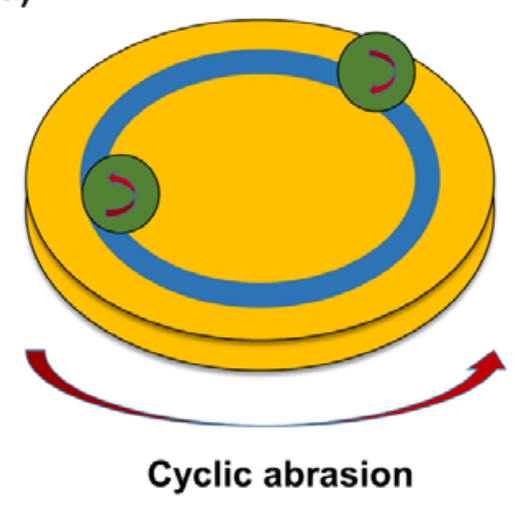

(b)

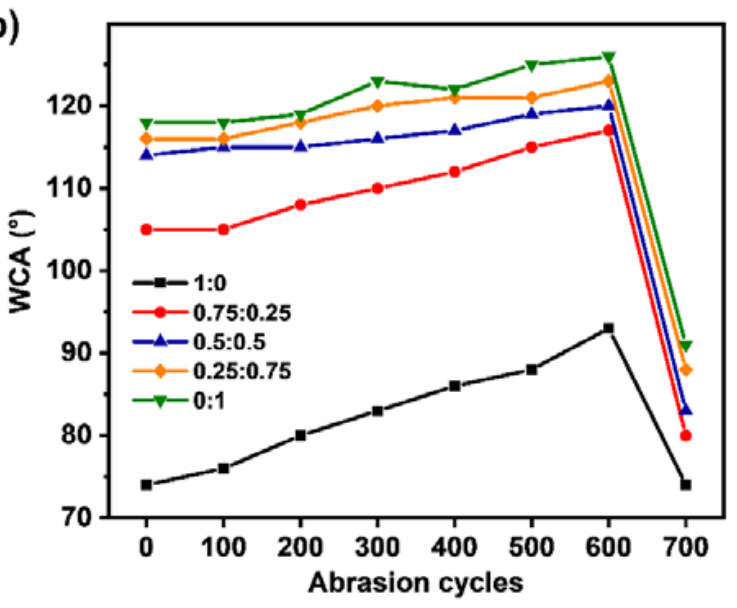

Figure 4. (a) Schematic illustration of abrasion test on a leather and (b) WCA change on the coated leather after repeated abrasion.

\subsection{Potential UV Degradation}

Long straight-chain fluorocarbon compounds are limited by their non-degradable and bioaccumulative properties, so the materials to be prepared should be environmentally friendly and degradable. In this section, the leather samples treated with our synthesizing FPU waterproofing agent were exposed to ultraviolet light, and their WCAs at different UV irradiation times are compared in Figure 5. During the continuous irradiation of $120 \mathrm{~h}$, the WCAs of FPU coatings decreased by about $15^{\circ}$ at the end, but all their WCAs were more than $90^{\circ}$, still showing the hydrophobicity. Different from the PU sample, which was completely terminated by ethanol, the leather samples decorated with the FPU waterproof agent could still achieve the hydrophobic effect, which proved that the FPU waterproof agent had better ultraviolet aging resistance and broad outdoor application prospect. Compared to that the introduction of heptadecafluorodecyl triethoxysilane (FAS), the WCA of the coating barely decreased after $600 \mathrm{~min}$ of irradiation, while the WCA of FPU waterproofing agent decreased gradually after continuous UV irradiation. Therefore, this indicated that the FPU waterproofing agent prepared in this research exhibited potential photocatalytic degradation behavior.

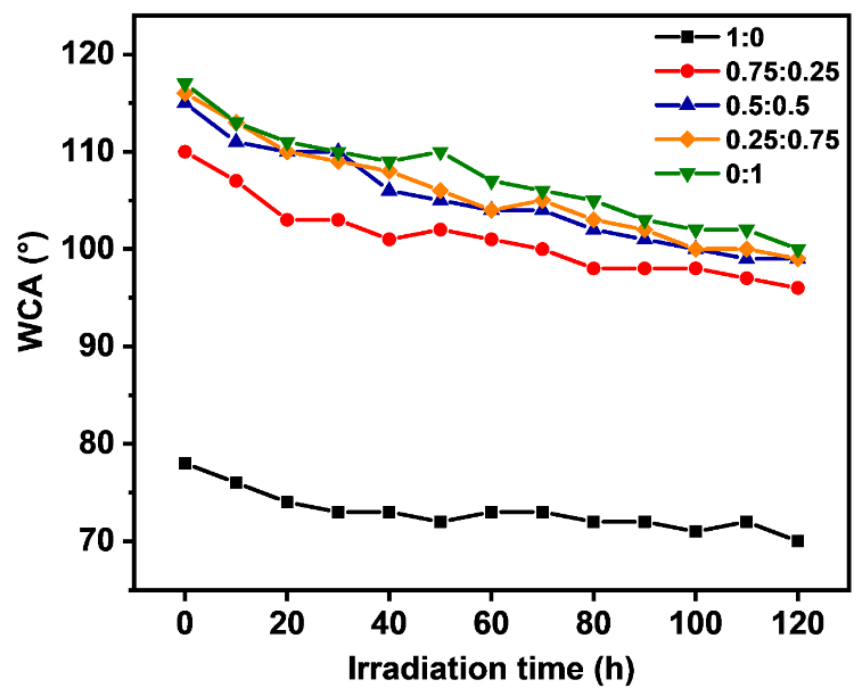

Figure 5. Change of WCA with UV irradiation time for waterproof coatings with different molar ratios of ET:FA. 
On the basis of the above test results and cost consideration, when the ET:FA molar ratio was $0.5: 0.5$, that is, when one end of a molecular chain was sealed with ethanol and the other end was sealed with fluoro-alcohol, we considered that the cost performance was the highest at this moment. Furthermore, we roll-coated this sample on the leather surface and comparatively tested the waterproofing properties of the FPU and the commercial C8 waterproofing agents. The correlative test results are described later.

\subsection{Waterproofing Grade}

As above discussion, the fluorine-containing chains enriched on the coating surface to make it waterproof. Figure $6(\mathrm{ia}-\mathrm{c})$ are the renderings of droplets of Grade 8 standard test solution on pure leather, the leather coated with the FPU, and the leather coated with the commercial C8 waterproofing agent, respectively. It is obvious that the two coated leather samples were not wetted after $10 \mathrm{~s}$ of placement. In consequence, the FPU waterproof agent embellishing leather had the waterproofing effect of Grade 8, almost one grade, as in the commercial C8 waterproofing agent below.

i

(a)

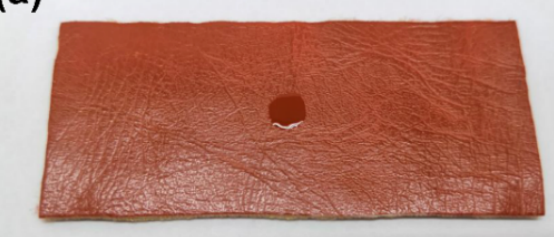

(b)

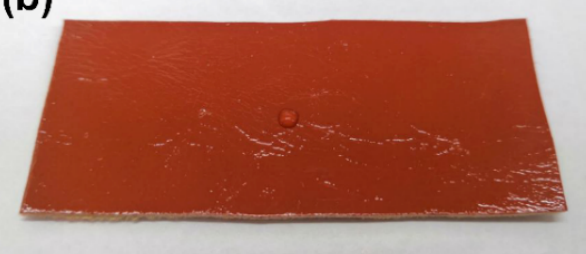

(c)

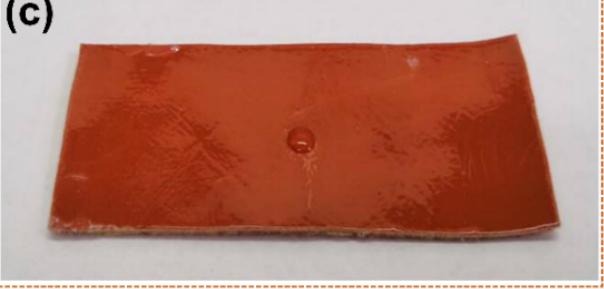

ii

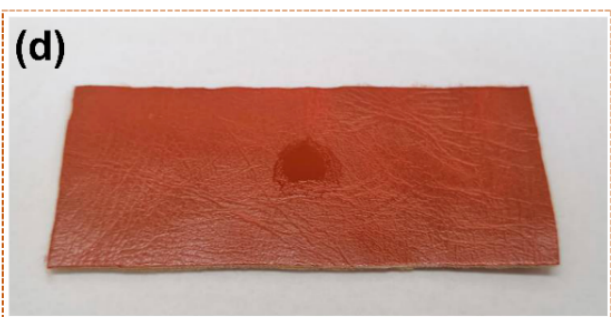

(e)

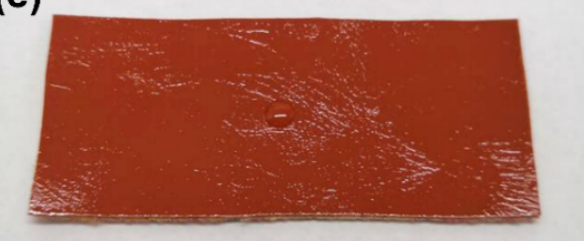

(f)

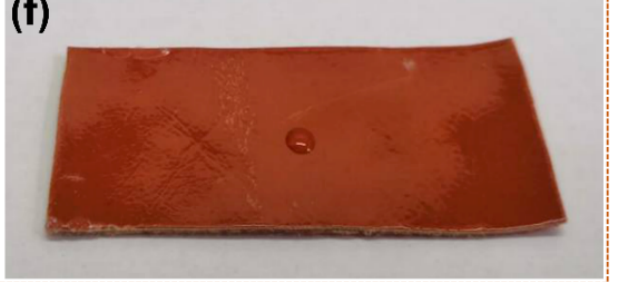

Figure 6. (i) Waterproofing grade test with 40/60 $(v / v)$ of water/isopropyl alcohol on uncoated leather (a), leather coated with FPU waterproofing agent (b), and commercial C8 waterproofing agent (c); (ii) Oilproofing grade test with n-heptane on uncoated leather (d), leather coated with FPU waterproofing agent (e), and commercial C8 waterproofing agent (f).

\subsection{Oilproofing Grade}

The FPU coating not only showed good waterproof properties but also special oilproof properties. Figure 6(iid-f) are the renderings of droplets of Grade 8 standard test solution on pure leather, the leather coated with the FPU, and the leather coated with the commercial C8 waterproofing agent, respectively. It can be clearly seen that the pure leather almost had no oil-proof performance, the test solution immediately wetted the leather, while the FPU modified leather had Grade 8 oil-proof effect, which was the same as that of the commercial C8 waterproofing agent. As a result, it could be used as a substitute for the commercial C8 waterproofing agent to develop its applications, showing a good market prospect. 


\subsection{Anti-Smudge Performance}

In real life, most materials are prone to losing some of their properties after suffering oil pollution, and so do many waterproof coatings. Thus, we investigated the anti-smudge performance of the FPU waterproofing agent modifying leather. Figure S1a,b respectively shows the initial dripping and $2 \mathrm{~s}$ sliding traces of ink droplets coated on the unmodified and FPU modified slant leather samples. The dark ink mark was expressly observed on the surface of uncoated leather, but the ink easily slipped off the FPU coated area without leaving a trace. Therefore, the incorporated fluorine-containing chain segments endowed the ink-repellant property so that the as-prepared material showed tremendous potential in anti-graffiti application.

\subsection{Solvent Resistance}

During testing the waterproofing property of FPU coating by using an isopropanol standard test solution (Figure 7i), we found that the commercial C8 waterproofing agent coating whitened after the test (Figure 7ia), while our prepared FPU waterproofing coating did not show any whitening phenomenon (Figure 7ib). Therefore, it is necessary to carry out solvent-resistant test (Figure 7ii).

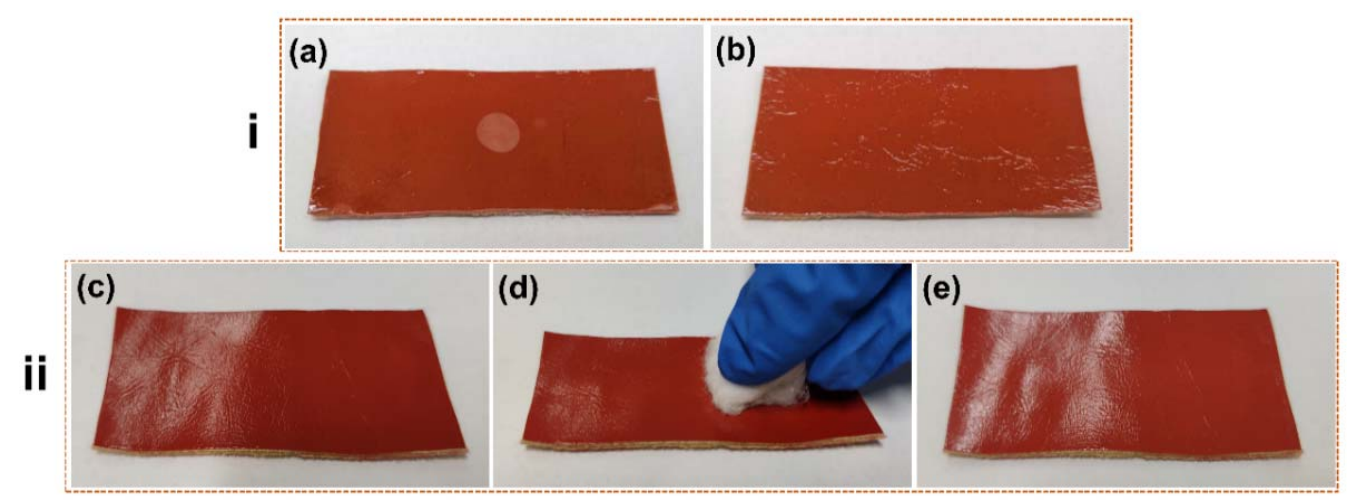

Figure 7. (i) Samples tested with isopropanol/water standard test solution: leathers coated with commercial C8 waterproofing agent (a) and FPU waterproofing agent (b); (ii) Solvent resistance test of the coating by hand wiping: (c) before wiping, (d) wiping action illustration, and (e) after wiping with the solvent.

It can be observed from Figure 7iie that the FPU coating after the repeated wiping with the standard test solvent showed no damage, the surface gloss was still retained, and the substrate was not exposed to the air at all. This experimental result proved that the FPU waterproofing agent prepared in this work had a superior resistance to organic solvent.

\section{Conclusions}

In conclusion, we successfully prepared an excellent comprehensive property of a novel leather waterproofing agent by introducing a short-branched fluoro-alcohol into polyurethane oligomer structure as the end-sealant. The investigation results showed that the waterproofing and oilproofing grades of the FPU waterproofing agent coating reached their highest (Grade 8) and the ink easily slipped without leaving a trace, indicating that the coating had excellent water-proof, oil-proof, and anti-smudge properties. Moreover, the coating could sustain the high abrasion resistance and exhibit potential photo-degradation after continuous UV irradiation of $120 \mathrm{~h}$. Compared with the commercial C8 waterproofing agent, the FPU coating also showed significant solvent resistance. The surface chemistry analysis revealed the real reason for this superior performance, which was attributed to the continuous increase in fluorine-containing chain segments with the increasing fluoroalcohol content and sub-sequent enrichment on the coating surface. This elaborately designed FPU waterproofing agent had many advantages in terms of either structure or property. This may avoid the straight-chain perfluoroalkyl compound (no less than C8) 
having the defined biotoxicity and environmental persistence. As a result, it is expected to be applied in various base materials. As for an investigation of the bioaccumulation of the FPU waterproofing agent, we will perform further research in a future work.

Supplementary Materials: The following are available online at https: / www.mdpi.com/article/ 10.3390/coatings11040395/s1, Table S1: Standard test liquids for waterproof grade test, Table S2: Standard test liquids for oilproof grade test, Figure S1: Snapshots of sliding ink droplets on uncoated leather (a) and leather coated with FPU waterproofing agent (b).

Author Contributions: Conceptualization, S.S.; methodology, S.S.; software, C.L.; formal analysis, J.Y.; investigation, Z.P.; resources, M.P.; writing—original draft preparation, S.S.; writing—review and editing, M.P.; visualization, S.S.; supervision, J.W. All authors have read and agreed to the published version of the manuscript.

Funding: Please add: This research was funded by the National Natural Science Foundation of China (No. 51973050) and the Hebei Province Natural Science Fund (B2019202114).

Institutional Review Board Statement: Not applicable.

Informed Consent Statement: Not applicable.

Data Availability Statement: The data presented in this study are available on request from the corresponding author.

Acknowledgments: We acknowledge financial support for this work from the National Natural Science Foundation of China (No. 51973050) and the Hebei Province Natural Science Fund (B2019202114). Thanks to Xinwen Jin of Hefei Jinsheng Chemical Co. Ltd., Anhui, China, for his kind help and beneficial discussion of this study.

Conflicts of Interest: The authors declare no conflict of interest.

\section{References}

1. Wang, J.; Raza, A.; Si, Y.; Cui, L.; Ge, J.; Ding, B.; Yu, J. Synthesis of superamphiphobic breathable membranes utilizing SiO 2 nanoparticles decorated fluorinated polyurethane nanofibers. Nanoscale 2012, 4, 7549-7556. [CrossRef]

2. Wu, Z.; Tang, L.; Dai, J.; Qu, J. Synthesis and properties of fluorinated non-isocyanate polyurethanes coatings with good hydrophobic and oleophobic properties. J. Coat. Technol. Res. 2019, 16, 1233-1241. [CrossRef]

3. Zhuge, W.; Pan, M.; Chang, Y.; Yuan, J.; Wang, X.; Sun, C.; Zhang, G. Monodispersed composite particles prepared via emulsifier-free emulsion polymerization using waterborne polyurethane as microreactors. J. Appl. Polym. Sci. 2014, $131,40985$. [CrossRef]

4. Khan, F.; Khan, A.; Tuhin, M.; Rabnawaz, M.; Li, Z.; Naveed, M. A novel dual-layer approach towards omniphobic polyurethane coatings. RSC Adv. 2019, 9, 26703-26711. [CrossRef]

5. Zheng, H.; Pan, M.; Wen, J.; Yuan, J.; Zhu, L.; Yu, H. Robust, transparent, and superhydrophobic coating fabricated with waterborne polyurethane and inorganic nanoparticle composites. Ind. Eng. Chem. Res. 2019, 58, 8050-8060. [CrossRef]

6. Mao, X.; Chen, Y.; Si, Y.; Li, Y.; Wan, H.; Yu, J.; Sun, G.; Ding, B. Novel fluorinated polyurethane decorated electrospun silica nanofibrous membranes exhibiting robust waterproof and breathable performances. RSC Adv. 2013, 3, 7562-7569. [CrossRef]

7. Jiang, Y.; Li, L.; Wang, H.; Wang, R.; Tian, Q. Influence of acrylic emulsion on polymer-cement waterproof coating. Adv. Mat. Res. 2015, 1129, 263-269. [CrossRef]

8. Huang, J.; Meng, W.; Qing, F. Synthesis and repellent properties of vinylidene fluoride-containing polyacrylates. J. Fluor. Chem. 2007, 128, 1469-1477. [CrossRef]

9. Chen, L.; Shi, H.; Wu, H.; Xiang, J. Preparation and characterization of a novel fluorinated acrylate resin. J. Fluor. Chem. 2010, 131, 731-737. [CrossRef]

10. Wang, J.; Chen, X.; Kang, Y.; Yang, G.; Yu, L.; Zhang, P. Preparation of superhydrophobic poly (methyl methacrylate)-silicon dioxide nanocomposite films. Appl. Surf. Sci. 2010, 257, 1473-1477. [CrossRef]

11. Li, Q.; Fan, Z.; Chen, C.; Li, Z. Water and oil repellent properties affected by the crystallinity of fluorocarbon chain in fluorinesilicon containing finishing agent. J. Text. Inst. 2020, 111, 360-369. [CrossRef]

12. Pan, H.; Li, G. Emulsion waterproof agent and its effects on intrinsic properties of gypsum. Asian J. Chem. 2013, 25, 5042-5046. [CrossRef]

13. Zhou, Y.; Liu, C.; Gao, J.; Chen, Y.; Yu, F.; Chen, M.; Zhang, H. A novel hydrophobic coating film of water-borne fluoro-silicon polyacrylate polyurethane with properties governed by surface self-segregation. Prog. Org. Coat. 2019, 134, 134-144. [CrossRef]

14. Wehbi, M.; Banerjee, S.; Mehdi, A.; Alaaeddine, A.; Hachem, A.; Ameduri, B. Vinylidene fluoride-based polymer network via cross-linking of pendant triethoxysilane functionality for potential applications in coatings. Macromolecules 2017, 50, 9329-9339. [CrossRef] 
15. Wang, C.; Li, X.; Du, B.; Li, P.; Lai, X.; Niu, Y. Preparation and properties of a novel waterborne fluorinated polyurethane-acrylate hybrid emulsion. Colloid Polym. Sci. 2014, 292, 579-587. [CrossRef]

16. Lin, J.; Jiang, F.; Wen, J.; Lv, W.; Porteous, N.; Deng, Y.; Sun, Y. Fluorinated and un-fluorinated N-halamines as antimicrobial and biofilm-controlling additives for polymers. Polymer 2015, 68, 92-100. [CrossRef] [PubMed]

17. Zhang, L.; Li, Y.; Yu, J.; Ding, B. Fluorinated polyurethane macroporous membranes with waterproof, breathable and mechanical performance improved by lithium chloride. RSC Adv. 2015, 5, 79807-79814. [CrossRef]

18. Li, N.; Zeng, F.; Wang, Y.; Qu, D.; Zhang, C.; Li, J.; Bai, Y. Synthesis and characterization of fluorinated polyurethane containing carborane in the main chain: Thermal, mechanical and chemical resistance properties. Chin. J. Polym. Sci. 2018, 36, 85-97. [CrossRef]

19. Zhang, L.; Kong, Q.; Kong, F.; Liu, T.; Qian, H. Synthesis and surface properties of novel fluorinated polyurethane base on F-containing chain extender. Polym. Adv. Technol. 2020, 31, 616-629. [CrossRef]

20. Yu, F.; Gao, J.; Liu, C.; Chen, Y.; Zhong, G.; Hodges, C.; Chen, M.; Zhang, H. Preparation and UV aging of nano-SiO $2 /$ fluorinated polyacrylate polyurethane hydrophobic composite coating. Prog. Org. Coat. 2020, 141, 105556. [CrossRef]

21. Zhao, D.; Pan, M.; Yuan, J.; Liu, H.; Song, S.; Zhu, L. A waterborne coating for robust superamphiphobic surfaces. Prog. Org. Coat. 2020, 138, 105368. [CrossRef]

22. Liu, C.; Liao, X.; Shao, W.; Liu, F.; Ding, B.; Ren, G.; Chu, Y.; He, J. Hot-melt Adhesive Bonding of Polyurethane/Fluorinated Polyurethane/Alkylsilane-Functionalized Graphene Nanofibrous Fabrics with Enhanced Waterproofness. Polymers 2020, 12, 836. [CrossRef]

23. Rabnawaz, M.; Liu, G. Graft-copolymer-based approach to clear, durable, and anti-smudge polyurethane coatings. Angew. Chem. Int. Edit. 2015, 54, 6516-6520. [CrossRef] [PubMed]

24. Ge, Z.; Zhang, X.; Dai, J.; Li, W.; Luo, Y. Synthesis, characterization and properties of a novel fluorinated polyurethane. Eur. Polym. J. 2009, 45, 530-536. [CrossRef]

25. Fu, K.; Lu, C.; Liu, Y.; Zhang, H.; Zhang, B.; Zhang, H.; Zhou, F.; Zhu, Q.; Zhu, B. Mechanically robust, self-healing superhydrophobic anti-icing coatings based on a novel fluorinated polyurethane synthesized by a two-step thiol click reaction. Chem. Eng. J. 2021, 404, 127110. [CrossRef]

26. Zhao, J.; Li, Y.; Sheng, J.; Wang, X.; Liu, L.; Yu, J.; Ding, B. Environmentally friendly and breathable fluorinated polyurethane fibrous membranes exhibiting robust waterproof performance. ACS Appl. Mater. Interfaces 2017, 9, 29302-29310. [CrossRef]

27. Jeong, H.Y.; Lee, M.H.; Kim, B.K. Surface modification of waterborne polyurethane. Colloids and Surfaces A: Physicochemical and Engineering Aspects. Colloids Surf. A 2006, 290, 178-185. [CrossRef]

28. Chen, K.; Kuo, J. Synthesis and properties of novel fluorinated aliphatic polyurethanes with fluoro chain extenders. Macromol. Chem. Phys. 2000, 201, 2676-2686. [CrossRef]

29. Tan, H.; Guo, M.; Du, R.; Xie, X.; Li, J.; Zhong, Y.; Fu, Q. The effect of fluorinated side chain attached on hard segment on the phase separation and surface topography of polyurethanes. Polymer 2004, 45, 1647-1657. [CrossRef]

30. Wang, L. Experimental and theoretical characterization of the morphologies in fluorinated polyurethanes. Polymer 2007, 48, 894-900. [CrossRef]

31. Zhao, J.; Wang, X.; Liu, L.; Yu, J.; Ding, B. Human skin-like, robust waterproof, and highly breathable fibrous membranes with short perfluorobutyl chains for eco-friendly protective textiles. ACS Appl. Mater. Interfaces 2018, 10, 30887-30894. [CrossRef]

32. Li, S.; Huang, J.; Ge, M.; Li, S.; Xing, T.; Chen, G.; Liu, Y.; Zhang, K.; Al-Deyab, S.; Lai, Y. Controlled grafting superhydrophobic cellulose surface with environmentally-friendly short fluoroalkyl chains by ATRP. Mater. Des. 2015, 85, 815-822. [CrossRef]

33. Soto, D.; Ugur, A.; Farnham, T.A.; Gleason, K.K.; Varanasi, K.K. Short-Fluorinated iCVD Coatings for Nonwetting Fabrics. Adv. Funct. Mater. 2018, 28, 1707355. [CrossRef]

34. Jiang, J.; Zhang, G.; Wang, Q.; Zhang, Q.; Zhan, X.; Chen, F. Novel fluorinated polymers containing short perfluorobutyl side chains and their super wetting performance on diverse substrates. ACS Appl. Mater. Interfaces 2016, 8, 10513-10523. [CrossRef] [PubMed]

35. Rodea-Palomares, I.; Leganés, F.; Rosal, R.; Fernández-Pinas, F. Toxicological interactions of perfluorooctane sulfonic acid (PFOS) and perfluorooctanoic acid (PFOA) with selected pollutants. J. Hazard. Mater. 2012, 201, 209-218. [CrossRef] [PubMed]

36. Gallo, V.; Leonardi, G.; Genser, B.; Lopez-Espinosa, M.J.; Frisbee, S.J.; Karlsson, L.; Ducatman, A.M.; Fletcher, T. Serum perfluorooctanoate (PFOA) and perfluorooctane sulfonate (PFOS) concentrations and liver function biomarkers in a population with elevated PFOA exposure. Environ. Health Perspect. 2012, 120, 655-660. [CrossRef]

37. Yang, Y.; Shen, J.; Zhang, L.; Li, X. Preparation of a novel water and oil-repellent fabric finishing agent containing a short perfluoroalkyl chain and its application in textiles. Mater. Res. Innov. 2015, 19, 401-404. [CrossRef]

38. Zhang, Q.; Wang, Q.; Jiang, J.; Zhan, X.; Chen, F. Microphase structure, crystallization behavior, and wettability properties of novel fluorinated copolymers poly (perfluoroalkyl acrylate-co-stearyl acrylate) containing short perfluorohexyl chains. Langmuir 2015, 31, 4752-4760. [CrossRef]

39. Zhang, D.; Sha, M.; Pan, R.; Lin, X.; Xing, P.; Jiang, B. Synthesis and properties study of novel fluorinated surfactants with perfluorinated branched ether chain. J. Fluor. Chem. 2019, 219, 62-69. [CrossRef]

40. Jin, H.; Xu, W.; Dai, J. Applications of Hexafluoropropene Trimer. Zhejiang Chem. Ind. 2016, 47, 1-8.

41. Wen, J.; Jia, Z.; Zhang, X.; Pan, M.; Yuan, J.; Zhu, L. Tough, thermo-Responsive, biodegradable and fast self-healing polyurethane hydrogel based on microdomain-closed dynamic bonds design. Mater. Today Commun. 2020, 25, 101569. [CrossRef] 
42. Zhao, J.; Zhou, T.; Zhang, J.; Chen, H.; Yuan, C.; Zhang, W.; Zhang, A. Synthesis of a waterborne polyurethane-fluorinated emulsion and its hydrophobic properties of coating films. Ind. Eng. Chem. Res. 2014, 53, 19257-19264. [CrossRef]

43. Xue, D.; Wang, X.; Ni, H.; Zhang, W.; Xue, G. Surface segregation of fluorinated moieties on random copolymer films controlled by random-coil conformation of polymer chains in solution. Langmuir 2009, 25, 2248-2257. [CrossRef] [PubMed] 